Ark. Mat., 42 (2004), 153-171

(C) 2004 by Institut Mittag-Leffler. All rights reserved

\title{
Multiple summing operators on $C(K)$ spaces
}

\author{
David Pérez-García and Ignacio Villanueva $\left({ }^{1}\right)$
}

\begin{abstract}
In this paper, we characterize, for $1 \leq p<\infty$, the multiple $(p, 1)$-summing multilinear operators on the product of $C(K)$ spaces in terms of their representing polymeasures. As consequences, we obtain a new characterization of $(p, 1)$-summing linear operators on $C(K)$ in terms of their representing measures and a new multilinear characterization of $\mathcal{L}_{\infty}$ spaces. We also solve a problem stated by M. S. Ramanujan and E. Schock, improve a result of H. P. Rosenthal and S. J. Szarek, and give new results about polymeasures.
\end{abstract}

\section{Introduction and notation}

Motivated by the importance of the theory of absolutely summing linear operators, there have been some attempts to generalize this concept and the related results and tools to the multilinear setting. Most of the previous efforts in this direction use the following definition of multilinear $\left(q: p_{1}, \ldots, p_{n}\right)$-summing operator, for certain choices of $q$ and $p_{i}$ :

A multilinear operator $T: X_{1} \times \ldots \times X_{n} \rightarrow Y$ is called $\left(q: p_{1}, \ldots, p_{n}\right)$-summing if there exists a constant $K>0$ such that

$$
\left(\sum_{i=1}^{m}\left\|T\left(x_{i}^{1}, \ldots, x_{i}^{n}\right)\right\|^{q}\right)^{1 / q} \leq K \prod_{j=1}^{n}\left\|\left(x_{i}^{j}\right)_{i=1}^{m}\right\|_{p_{j}}^{w_{j}}
$$

for all choices of $m \in \mathbf{N}$ and $x_{1}^{j}, \ldots, x_{m}^{j} \in X_{j}$.

The interested reader can consult [9]: [19] or [22] and the references therein to know more about this class of operators.

Recently, F. Bombal and both authors in [5] and [24]: and M. C. Matos in [20] have defined and studied the class of multiple summing multilinear operators, see Definition 2.1 (although the origin of this class goes back to [27]). This class extends the notion of $p$-summing operator to the multilinear setting in a different

(1) Both authors were partially supported by DGICYT grant BMF2001-1284. 
way, it behaves better in many ways than the previous definitions of $p$-summing multilinear operators, and seems to be the "right" generalization of the linear case for many applications.

In particular, we prove in [5]. [23]. [24] and [25] several multilinear generalizations of Grothendieck's theorem and relations with nuclear and Hilbert-Schmidt multilinear operators that extend and generalize classical linear results. It is easy to see that this "good behavior" is not shared by the $\left(q: p_{1} \ldots \ldots p_{n}\right)$-summing operators defined as above.

In this paper we continue studying the multiple summing multilinear operators. We give a simple characterization of the multiple 1-summing operators and the muitiple $(p, 1)$-summing operators on the product of $C(K)$ spaces in terms of their representing polymeasure. As a particular case, we obtain a new characterization of $(p, 1)$-summing operators defined on $C(K)$ spaces in terms of their representing measure. As an application we can prove the rather surprising Corollary 3.2. This corollary will be the main tool used in Proposition 3.4. where we improve a result of H. P. Rosenthal and S. J. Szarek. Another application of our results is Proposition 3.6, which gives a multilinear characterization of $\mathcal{L}_{x}$ spaces related to the main result of [9].

Several results in this paper (particularly Theorem 2.2 and Proposition 3.1) show that the class of multiple $p$-summing multilinear operators is relatively "small". Thus, these results are especially surprising when compared with the Grothendieck type theorems given in [5] which show that every multilinear operator from the product of $\mathcal{L}_{\infty}$ spaces to an $\mathcal{L}_{1}$ space is multiple 2 -summing, and that every multilinear operator from the product of $\mathcal{L}_{1}$ spaces to a Hilbert space is multiple 1-summing.

In addition, we use some results of [5] to establish Example 3.13, which solves a problem stated in $[27]$, and also to give non-trivial new results about polymeasures (Corollaries 3.18 and 3.21 ).

The notation and terminology used throughout the paper are standard in Banach space theory, as for instance in [12]. This book is also our main reference for basic facts, definitions and unexplained notation throughout the paper. However, before going any further, we shall establish some terminology: $\mathbf{K}$ will be the scalar field, which can be considered to be either the real or complex numbers; $X_{i}$ and $Y$ will always be Banach spaces; and $\mathcal{L}(X . Y)$ will denote the Banach space of bounded linear mappings from $X$ to $Y$. For $n \geq 2 . \mathcal{L}^{n}\left(X_{1} \ldots, X_{n}: Y\right)$ will be the Banach space of all the continuous $n$-linear mappings from $X_{1} \times \ldots \times X_{n}$ into $Y$. When $Y=\mathbf{K}$ we will omit it and. from now on. operator will mean linear or multilinear continuous mapping. As usual. $X_{1} \widehat{\bar{\varepsilon}}_{\Xi} \ldots \widehat{\bar{\varepsilon}}_{\bar{\varepsilon}} X_{n}$ stands for the (completion of the) injective tensor product of the Banach spaces $X_{1} \ldots . X_{n}$ and $X_{1} \widehat{\otimes}_{\pi} \ldots \widehat{\otimes}_{\pi} X_{n}$ will denote (the completion of) their projective tensor product. Given a Banach 
space $X, B_{X}$ denotes its unit ball, $X^{*}$ stands for its topological dual and $\omega^{*}$ for the weak-star topology in $X^{*}$.

Given $X, 1 \leq p<\infty$ and a finite sequence $\left(x_{i}\right)_{i=1}^{m} \subset X$, we let

$$
\left\|\left(x_{i}\right)_{i=1}^{m}\right\|_{p}^{\omega}=\sup \left\{\left(\sum_{i=1}^{m}\left|\left\langle x^{*}, x_{i}\right\rangle\right|^{p}\right)^{1 / p}: x^{*} \in B_{X^{*}}\right\} .
$$

For $1 \leq p \leq q<\infty$, we write $\Pi_{(q . p)}(X, Y)$ for the Banach space of $(q, p)$-summing operators from $X$ into $Y$, and $\pi_{(q \cdot p)}(T)$ stands for the $(q . p)$-summing norm of $T \in \Pi_{(q, p)}(X, Y)$. When $q=p$ we have the $p$-summing operators, and the notation will then be $\Pi_{p}(X, Y)$ and $\pi_{p}(T)$.

Let $1 \leq p \leq \infty$ and $\lambda>1$. A Banach space $X$ is said to be an $\mathcal{L}_{p . \lambda}$ space if for every finite-dimensional subspace $E \subset X$ there exists another finite-dimensional subspace $F$, with $E \subset F \subset X$ and such that there exists an isomorphism $v: F \rightarrow l_{p}^{\operatorname{dim} F}$ with $\|v\|\left\|v^{-1}\right\|<\lambda$. We say that $X$ is an $\mathcal{L}_{p}$ space if it is an $\mathcal{L}_{p . \lambda}$ space for some $\lambda>1$. Clearly, $L_{p}(\mu)$ is the basic example of an $\mathcal{L}_{p}$-space.

Given $n, m_{1}, \ldots, m_{n} \in \mathbf{N},\left(x_{i_{1} \ldots, i_{n}}\right)_{i_{1} \ldots \ldots i_{n}=1}^{m_{1} \ldots \ldots m_{n}}$ denotes a multiindex sequence with the index $i_{j}$ varying from 1 to $m_{j}, 1 \leq j \leq n$.

If $T: X_{1} \times \ldots \times X_{n} \rightarrow Y$ is a multilinear operator, we write $A B(T): X_{1}^{* *} \times \ldots \times$ $X_{n}^{* *} \rightarrow Y^{* *}$ for its so-called Aron-Berner extension. which in general is not unique (see [3], or [8] and the references therein, for basic facts and different equivalent formulations of the Aron-Berner extension).

Let $\Sigma_{j}$ be the Borel $\sigma$-algebra of a compact space $K_{j}, 1 \leq j \leq n$ (or. in general. a $\sigma$-algebra defined on a set $\Omega_{j}$ ). A function $\eta_{i}: \Sigma_{1} \times \ldots \times \Sigma_{n} \longrightarrow Y$ is a (countably additive) polymeasure if it is separately (countably) additive.

Given a polymeasure $\gamma: \Sigma_{1} \times \ldots \times \Sigma_{n} \rightarrow Y$. as in the case $n=1$, its semivariation is defined as the set function

$$
\|\gamma\|: \Sigma_{1} \times \ldots \times \Sigma_{n} \longrightarrow[0 .+x]
$$

given by

$$
\|\gamma\|\left(A_{1}, \ldots, A_{n}\right)=\sup \left\|\sum_{k_{1}=1}^{r_{1}} \ldots \sum_{k_{n}=1}^{r_{n}} a_{k_{1}}^{1} \ldots a_{k_{n}}^{n} \gamma\left(A_{k_{1}}^{1} \ldots . A_{k_{n}}^{n}\right)\right\|
$$

where the supremum is taken over all the finite $\sum_{j}$-partitions $\left(A_{k_{j}}^{j}\right)_{k_{j}=1}^{r_{j}}$ of $A_{j}$. $1 \leq j \leq n$, and all the collections $\left(a_{k_{j}}^{j}\right)_{k_{j}=1}^{r_{j}}$ in the unit ball of the scalar field.

Let us also recall that its variation is defined as the set function

$$
v(\gamma): \Sigma_{1} \times \ldots \times \Sigma_{n} \longrightarrow[0,+\infty]
$$


given by

$$
v(\gamma)\left(A_{1}, \ldots, A_{n}\right)=\sup \sum_{k_{1}=1}^{r_{1}} \ldots \sum_{k_{n}=1}^{r_{n}}\left\|\gamma_{i}\left(A_{k_{1}}^{1}, \ldots, A_{k_{n}}^{n}\right)\right\|,
$$

where the supremum is taken over all the finite $\Sigma_{j}$-partitions $\left(A_{k_{j}}^{j}\right)_{k_{j}=1}^{r_{j}}$ of $A_{j}$, $1 \leq j \leq n$.

In general, given $1 \leq p<\infty$, we can define its $p$-variation as the set function

$$
v_{p}(\gamma): \Sigma_{1} \times \ldots \times \Sigma_{n} \rightarrow[0,+\infty]
$$

given by

$$
v_{p}(\gamma)\left(A_{1}, \ldots, A_{n}\right)=\sup \left(\sum_{k_{1}=1}^{r_{1}} \ldots \sum_{k_{n}=1}^{r_{n}}\left\|\gamma\left(A_{k_{1}}^{1}, \ldots, A_{k_{n}}^{n}\right)\right\|^{p}\right)^{1 / p}
$$

where the supremum is again taken over all the finite $\Sigma_{j}$-partitions $\left(A_{k_{j}}^{j}\right)_{k_{j}=1}^{r_{j}}$ of $A_{j}$, $1 \leq j \leq n$.

If $\gamma$ has finite semivariation, an elementary integral $\int\left(f_{1}, f_{2}, \ldots, f_{n}\right) d \gamma$ can be defined, where $f_{j}$ are bounded $\Sigma_{j}$-measurable scalar functions, just taking the limit of the integrals of $n$-tuples of simple functions (with the obvious definition) uniformly converging to the $f_{j}$ 's.

If $K_{1}, \ldots, K_{n}$ are compact Hausdorff spaces, then every multilinear operator $T \in$ $\mathcal{L}^{n}\left(C\left(K_{1}\right), \ldots, C\left(K_{n}\right) ; Y\right)$ has a unique representing polymeasure $\gamma: \Sigma_{1} \times \ldots \times \Sigma_{n} \rightarrow$ $Y^{* *}$ with finite semivariation, in such a way that

$$
T\left(f_{1}, \ldots, f_{n}\right)=\int\left(f_{1}, \ldots, f_{n}\right) d \gamma \text { for } f_{j} \in C\left(K_{j}\right)
$$

and such that for every $y^{*} \in Y^{*}, y^{*} \approx$ is a separately regular countably additive scalar polymeasure. The idea behind this representation theorem can be easily described.

Given a compact Hausdorff space and its Borel $\sigma$-algebra $\Sigma$, we write $B(\Sigma)$ for the completion under the supremum norm of the space $S(\Sigma)$ of the $\Sigma$-simple scalar valued functions. It is well known that $C(K) \stackrel{1}{\hookrightarrow} B(\Sigma) \stackrel{1}{\hookrightarrow} C(K)^{* *}$ : where $\stackrel{1}{\hookrightarrow}$ denotes isometric embedding. So, for the operator $T$ we consider its Aron-Berner extension to the product of the biduals $A B(T)$ (which is unique in this case) and restrict it to $\bar{T}: B\left(\Sigma_{1}\right) \times \ldots \times B\left(\Sigma_{n}\right) \rightarrow Y^{* *}$. Now we define $\gamma\left(A_{1}, \ldots, A_{n}\right)=\bar{T}\left(\chi_{A_{1}}, \ldots, \chi_{A_{n}}\right)$. In fact, as for the case of $C(K)$ spaces, simple reasonings yield an isometric isomorphism between $\mathcal{L}^{n}\left(B\left(\Sigma_{1}\right), \ldots, B\left(\Sigma_{n}\right) ; Y\right)$ and $\operatorname{bpm}\left(\Sigma_{1} \ldots, \Sigma_{n}: Y\right)$, the Banach space of the polymeasures with bounded semivariation defined on $\Sigma_{1} \times \ldots \times \Sigma_{n}$ with values in $Y$, endowed with the semivariation norm (see [6] and the references therein for more information about polymeasures and the representation theorem). 


\section{Definition and first results}

We start by recalling our definition.

Definition 2.1. Let $1 \leq p_{1}, \ldots, p_{n} \leq q<+\infty$. A multilinear operator $T: X_{1} \times \ldots \times$ $X_{n} \rightarrow Y$ is multiple $\left(q ; p_{1}, \ldots, p_{n}\right)$-summing, if there exists a constant $K>0$ such that, for every choice of sequences $\left(x_{i_{j}}^{j}\right)_{i_{j}=1}^{m_{j}} \subset X_{j}$ the following relation holds

$$
\left(\sum_{i_{1}=1}^{m_{1}} \ldots \sum_{i_{n}=1}^{m_{n}}\left\|T\left(x_{i_{1}}^{1}, \ldots, x_{i_{n}}^{n}\right)\right\|^{q}\right)^{1 / q} \leq K \prod_{j=1}^{n}\left\|\left(x_{i_{j}}^{j}\right)_{i_{j}=1}^{m_{j}}\right\|_{p_{j}}^{w_{j}} .
$$

In that case, we define the multiple $\left(q ; p_{1}, \ldots, p_{n}\right)$-summing norm of $T$ by

$$
\pi_{\left(q ; p_{1} \ldots, p_{n}\right)}(T)=\min \{K: K \text { satisfies }(1)\}
$$

A multiple $(q ; p, \ldots, p)$-summing operator will be called multiple $(q, p)$-summing. and we write $\pi_{(q ; p)}$ for the associated norm. Moreover, a multiple $(p, p)$-summing operator will be called multiple $p$-summing and we write $\pi_{p}$ for the associated norm. The class $\Pi_{\left(q ; p_{1}, \ldots, p_{n}\right)}^{n}\left(X_{1}, \ldots, X_{n} ; Y\right)$ of multiple $\left(q: p_{1}, \ldots, p_{n}\right)$-summing multilinear operators is easily seen to be a Banach space with its norm $\pi_{\left(q: p_{1} \ldots \ldots p_{n}\right)}$.

As in the linear case, if there exists $1 \leq j \leq n$ such that $p_{j}>q$, only the zero operator can satisfy (1). This is the reason to introduce the hypothesis $1 \leq p_{1}, \ldots, p_{n} \leq$ $q<+\infty$. Let us start showing the most basic example of this class of operators. Let $T: X_{1} \times \ldots \times X_{n} \rightarrow Y$ be a multilinear operator. Suppose that $T$ is continuous in the $\varepsilon$ topology and that its linearization $\widehat{T}: X_{1} \widehat{\otimes}_{\varepsilon} \ldots \widehat{\otimes}_{\varepsilon} X_{n} \rightarrow Y$ is $(q, p)$ summing. Then, it follows easily from the definitions that $T$ is multiple $(q, p)$ summing. In particular, for any $x_{j}^{*} \in X_{j}^{*}$, the multilinear form $x_{1}^{*} \gtrless \notin x_{n}^{*}$ defined by $\left(x_{1}^{*} \otimes \ldots \otimes x_{n}^{*}\right)\left(x_{1}, \ldots, x_{n}\right)=x_{1}^{*}\left(x_{1}\right) \ldots x_{n}^{*}\left(x_{n}\right)$ is multiple $(q, p)$-summing for any $1 \leq p \leq q \leq \infty$. It is probably worth mentioning that. in general, multilinear forms need not be multiple $p$-summing, as follows from Propositions 3.1 and [20].

Note that in this definition we require the sum

$$
\left(\sum_{i_{1}=1}^{m_{1}} \ldots \sum_{i_{n}=1}^{m_{n}}\left\|T\left(x_{i_{1}}^{1}, \ldots, x_{i_{n}}^{n_{n}}\right)\right\|^{q}\right)^{1 / q}
$$

to be controlled by the product $\prod_{j=1}^{n}\left\|\left(x_{i_{j}}^{j}\right)_{i_{j}=1}^{m_{j}}\right\|_{p_{j}}^{\omega}$, whereas in the definition of $\left(q ; p_{1}, \ldots, p_{n}\right)$-summing operators mentioned in the introduction and used previously by other authors, it is the "diagonal" sum

$$
\left(\sum_{i=1}^{m}\left\|T\left(x_{i}^{1}, \ldots, x_{i}^{n}\right)\right\|^{q}\right)^{1 / q}
$$


that must be controlled by the same product.

We show first the good behavior with respect to the extensions to the bidual that our operators share with the $(q . p)$-summing linear operators. Recall that the Aron-Berner extension of a multilinear operator is. in many ways, the natural generalization of the bitranspose of a linear operator. In that sense, the notion of weakly compact linear operator extends to the notion of multilinear operator whose Aron-Berner extension remains in the image space. Following exactly the steps given in the proof of [14, Theorem 2.2] we obtain the following result.

Theorem 2.2. Let $T: X_{1} \times \ldots \times X_{n} \rightarrow Y$ be a multiple $p$-summing multilinear operator. Then its Aron-Berner extension $\mathrm{AB}(T)$ belongs to $\mathcal{L}\left(X_{1}^{* *}, \ldots, X_{n}^{* *} ; Y\right)$.

We also have the following result which we will need later.

Theorem 2.3. Let $1 \leq p_{1}, \ldots, p_{n} \leq q<\infty$. A multilinear operator $T: X_{1} \times \ldots \times$ $X_{n} \rightarrow Y$ is multiple $\left(q: p_{1}, \ldots, p_{n}\right)$-summing if and only if its Aron-Berner extension is multiple $\left(q ; p_{1}, \ldots, p_{n}\right)$-summing.

Moreover, in that case

$$
\pi_{\left(q: p_{1} \ldots . p_{n}\right)}(T)=\pi_{\left(q: p_{1} \ldots . p_{n}\right)}(\mathrm{AB}(T))
$$

The proof is obvious once we prove the following lemma.

Lemma 2.4. Let $X$ be a Banach space. $n \in \mathbf{N}$ and $1 \leq p<\infty$. Let $\left(z_{i}\right)_{i=1}^{m} \subset X^{* *}$. Then there exist a directed set $\Omega$ and nets $\left(x_{\alpha}^{i}\right)_{\alpha \in \Omega} \subset X$ such that

$$
x_{\alpha}^{i} \stackrel{\omega^{*}}{\longrightarrow} z_{i} \text { for every } 1 \leq i \leq n
$$

and such that

$$
\left\|\left(x_{\alpha}^{i}\right)_{i=1}^{m}\right\|_{p}^{\omega} \leq\left\|\left(z_{i}\right)_{i=1}^{m}\right\|_{p}^{\omega} \quad \text { for every } \alpha \in \Omega
$$

Proof. According to [11, Proposition 8.1], we know that the mapping given by $\left(y_{i}\right)_{i=1}^{m} \mapsto \sum_{i=1}^{m} e_{i} \otimes y_{i}$ establishes, for every Banach space $Y$, an isometric isomorphism between the Banach space of sequences of $m$ vectors of $Y$, endowed with the norm $\|\cdot\|_{p}^{\omega}$, and $l_{p}^{m} \otimes_{\varepsilon} Y$. Moreover the following isometric embeddings hold:

$$
l_{p}^{m} \otimes_{\varepsilon} X \hookrightarrow l_{p}^{m} \otimes_{\varepsilon} X^{* *} \hookrightarrow\left(l_{p}^{m} 8_{\varepsilon} X\right)^{* *}
$$

Since $\left(z_{i}\right)_{i=1}^{m} \subset l_{p}^{m} \otimes_{\varepsilon} X^{* *} \subset\left(l_{p}^{m} \otimes_{\varepsilon} X\right)^{* *}$, there exist a directed set $\Omega$ and a net $\left(w_{\alpha}\right)_{\alpha \in \Omega} \subset l_{p}^{m} \otimes_{\varepsilon} X$ such that

$$
w_{\alpha} \stackrel{\omega^{*}}{\longrightarrow}\left(z_{i}\right)_{i=1}^{m} \quad \text { and } \quad\left\|w_{\alpha}\right\| \leq\left\|\left(z_{i}\right)_{i=1}^{m}\right\|_{p}^{\omega}
$$


Let $x_{\alpha}^{i}$ be such that $w_{\alpha}=\sum_{i=1}^{m} e_{i} \& x_{\alpha}^{i}$. We have that

$$
\left\|\left(x_{\alpha}^{i}\right)_{i=1}^{m}\right\|_{p}^{\omega}=\left\|w_{\alpha}\right\| \leq\left\|\left(z_{i}\right)_{i=1}^{m}\right\|_{p}^{\omega}
$$

and that, for every $x^{*} \in X^{*}$;

$$
\left\langle x^{*}, x_{\alpha}^{i}\right\rangle=\left\langle e_{i}^{*} \otimes x^{*}, w_{\alpha}\right\rangle \underset{\alpha \in \Omega}{\longrightarrow}\left\langle e_{i}^{*} \otimes x^{*}, \sum_{k=1}^{m} e_{k} \& z_{k}\right\rangle=z_{i}
$$

The following proposition can be easily proved as [19. Proposition 2.5].

Proposition 2.5. Let $T: X_{1} \times \ldots \times X_{n} \rightarrow Y$ be a multilinear operator, let $1 \leq$ $k \leq n-1$ and let $T_{k}: X_{1} \times \ldots \times X_{k} \rightarrow \mathcal{L}^{n-k}\left(X_{k+1} \ldots . X_{n}: Y\right)$ be the associated $k$-linear operator.

If

$$
T_{k} \in \Pi_{\left(q ; p_{1} \ldots, p_{k}\right)}^{k}\left(X_{1}, \ldots, X_{k}: \Pi_{\left(q: p_{k+1} \ldots, p_{n}\right)}^{n-k}\left(X_{k+1} \ldots, X_{n}: Y\right)\right)
$$

then

$$
T \in \mathrm{I}_{\left(q ; p_{1}, \ldots, p_{n}\right)}^{n}\left(X_{1}, \ldots, X_{n} ; Y\right) \quad \text { and } \quad \pi_{\left(q ; p_{1} \ldots \ldots p_{n}\right)}(T) \leq \pi_{\left(q ; p_{1} \ldots \ldots p_{k}\right)}\left(T_{k}\right)
$$

We will see in Example 3.13 that, in general, the converse implication is not true. Nevertheless, it follows from Proposition 3.1 and [19] that the converse is true when $q=p_{1}=\ldots=p_{n}=1$ and all the $X_{j}$ are $C(K)$ spaces (or in general $\mathcal{L}_{x}$ spaces), or when $q=p_{1}=\ldots=p_{n}=2$ and all the $X_{j}$ and $Y$ are Hilbert spaces.

We state the following composition theorem for reference purposes, its proof, which can be seen in [5], follows along the lines of [12, 2.22].

Theorem 2.6. Let $u_{j} \in \Pi_{q}\left(X_{j}, Y_{j}\right)$ and $T \in \Pi_{p}^{n}\left(Y_{1} \ldots, Y_{n}: Z\right)$ and let $1 \leq r<+\infty$ be such that

$$
\frac{1}{r}=\frac{1}{p}+\frac{1}{q}
$$

Then $S=T\left(u_{1}, \ldots, u_{n}\right)$ is multiple $r$-summing and $\pi_{r}(S) \leq \pi_{p}(T) \prod_{j=1}^{n} \pi_{q}\left(u_{j}\right)$.

\section{The main results}

Given two Banach spaces $X$ and $Y$, we will denote by $I(X, Y)$ the space of integral linear operators from $X$ to $Y$. It is a Banach space with the integral norm $\|\cdot\|_{\text {int }}$ (see $[13$, p. 232] for the definitions). 
A multilinear operator $T \in \mathcal{L}^{n}\left(X_{1}, \ldots, X_{n}: Y\right)$ is integral if there exists a regular $Y^{* *}$-valued Borel measure $G$ of bounded variation on the product $B_{X_{1}^{*}} \times \ldots \times B_{X_{n}^{*}}$ such that

$$
T\left(x_{1}, \ldots, x_{n}\right)=\int_{B_{X_{1}^{*} \times \ldots \times B_{X_{n}^{*}}}} x_{1}^{*}\left(x_{1}\right) \ldots x_{n}^{*}\left(x_{n}\right) d G\left(x_{1}^{*}, \ldots, x_{n}^{*}\right)
$$

for all $\left(x_{1}, \ldots, x_{n}\right) \in X_{1} \times \ldots \times X_{n}$. The space $\mathcal{L}_{I}^{n}\left(X_{1} \ldots, X_{n}: Y\right)$ of integral multilinear operators is a Banach space with the norm $\|T\|_{\text {int }}=\inf \{v(G): G$ represents $T$ as above\}. These operators were defined in [30] (where they are called G-integral), although the definition is just a technical modification of a previous definition in [2]. In [30] it is proved that a multilinear operator $T: X_{1} \times \ldots \times X_{n} \rightarrow Y$ is integral if and only if its linearization $\hat{T}$ is continuous in the $\varepsilon$ topology and $\widehat{T}: X_{1} \widehat{\otimes}_{\varepsilon} \ldots \widehat{\otimes}_{\varepsilon} X_{n} \rightarrow Y$ is an integral operator. Moreover, in that case $\|T\|_{\text {int }}=\|\widehat{T}\|_{\text {int }}$.

We can now prove the following result.

Proposition 3.1. Let $K_{1}, \ldots, K_{n}$ be compact Hausdorff spaces, $T: C\left(K_{1}\right) \times \ldots \times$ $C\left(K_{n}\right) \rightarrow Y$ be a multilinear operator and let $\gamma$ be its representing polymeasure. Then the following are equivalent:

(i) $T$ is multiple 1-summing:

(ii) $v(\gamma)<\infty$;

(iii) $T$ is integral;

(iv) $T_{1} \in \Pi_{1}\left(C\left(K_{1}\right), \Pi_{1}\left(C\left(K_{2}\right), \ldots, \Pi_{1}\left(C\left(K_{n-1}\right), \Pi_{1}\left(C\left(K_{n}\right), Y\right)\right) \ldots\right)\right)$.

Moreover, in this case, all the norms coincide, i.e.

$$
\pi_{1}(T)=v(\gamma)=\|T\|_{\text {int }}=\pi_{1}\left(T_{1}\right)
$$

Proof. The implication (i) $\Rightarrow$ (ii) follows immediately from Theorem 2.3 and the fact that, if $(\Omega, \Sigma)$ is a measurable space and $\left(A_{i}\right)_{i=1}^{m}$ is a partition of $\Omega$, then the sequence $\left(\chi_{A_{i}}\right)_{i=1}^{m} \subset B(\Sigma)$ satisfies $\left\|\left(\chi_{A_{i}}\right)_{i=1}^{m}\right\|_{1}^{i} \leq 1$. The equivalence between (ii) and (iii) follows from [7. Corollary 4.2] and (iii) $\Rightarrow$ (iv) is a consequence of [30, Proposition 2.9]. Finally, (iv) $\Rightarrow$ (i) follows from Proposition 2.5.

As an immediate consequence we obtain the following very surprising result.

Corollary 3.2. Let $X_{j}, Y_{j}$ and $Z$ be Banach spaces, $1 \leq j \leq n$. Let $u_{j} \in$ $\Pi_{2}\left(X_{j}, Y_{j}\right)$ and $T \in \Pi_{2}^{n}\left(Y_{1}, \ldots, Y_{n}: Z\right)$. Then $S=T\left(u_{1}, \ldots, u_{n}\right)$ is integral and

$$
\|S\|_{\text {int }} \leq \pi_{2}(T) \prod_{j=1}^{n} \pi_{2}\left(u_{j}\right)
$$


Proof. It follows from the linear factorization theorem for 2-summing operators [12, Corollary 2.16] that there exist compact spaces $K_{j}$ and 2-summing operators $b_{j}: C\left(K_{j}\right) \rightarrow Y_{j}$ such that $u_{j}=b_{j} \circ i_{j}$, where $i_{j}: X_{j} \hookrightarrow C\left(K_{j}\right)$ are isometric inclusions, $1 \leq j \leq n$. Let us consider the operator $R=T\left(b_{1}, \ldots, b_{n}\right) \in \mathcal{L}^{n}\left(C\left(K_{1}\right), \ldots, C\left(K_{n}\right): Z\right)$. Applying Theorem 2.6 and Proposition 3.1 we get that $R$ is integral. Our result follows suit.

Remark 3.3. After the first version of this paper was written we have been able to prove that the operator $S$ in Corollary 3.2 is actually nuclear (see [25]).

We can apply this corollary to prove a proposition that improves one of the results in [29] (see the remark below). We will say that a Banach space $Y$ is a $G T$ space, or that $Y$ satisfies Grothendieck's theorem, if every linear operator from $Y$ to $l_{2}$ is 1-summing. According to Grothendieck's Theorem, $\mathcal{L}_{1}$ spaces are GT spaces, but there are several instances of GT spaces which are not $\mathcal{L}_{1}$-spaces, for example $L_{1} / H^{1}$ or the quotient of an $L_{1}$ space by a subspace isomorphic to a Hilbert space (see [26]). All the known examples of GT spaces have cotype 2: and it remains an open question whether this must always happen.

Proposition 3.4. For $1 \leq j \leq n$, let $X_{j}$ be an $\mathcal{L}_{\infty}$ space, $Y_{j}$ a $G T$ space with cotype 2 and $u_{j}: X_{j} \rightarrow Y_{j}$ a linear operator. Then, the operator

$$
u_{1} \otimes \ldots \otimes u_{n}: X_{1} \widehat{\otimes}_{\varepsilon} \ldots \widehat{\otimes}_{\varepsilon} X_{n} \longrightarrow Y_{1} \widehat{\otimes}_{\pi} \ldots \widehat{\otimes}_{\pi} Y_{n}
$$

is well defined and continuous.

Proof. By [7], it is sufficient to prove that, for every $T \in \mathcal{L}^{n}\left(Y_{1}, \ldots, Y_{n}\right)$, the composition $T\left(u_{1}, \ldots, u_{n}\right) \in \mathcal{L}_{I}^{n}\left(X_{1}, \ldots, X_{n}\right)$. It is shown in [5] that $T$ is multiple 2 -summing and, by [12, Theorem 11.14], $u_{j}$ is 2-summing for every $j$. Therefore, an appeal to Corollary 3.2 finishes the proof.

Remark 3.5. In [29], H. P. Rosenthal and S. J. Szarek mention that it would be desirable to determine pairs of (classes of) Banach spaces for which the conclusion of Proposition 3.4 holds. They obtained the result (in the case $n=2$ ) for $\mathcal{L}_{\infty}$ and $\mathcal{L}_{1}$ spaces. In that case, a direct proof can be given using induction. It is well known (see [15, Proposition 7] for a proof) that the projective tensor product of $\mathcal{L}_{1}$ spaces is an $\mathcal{L}_{1}$ space, and that the injective tensor product of $\mathcal{L}_{\infty}$ spaces is an $\mathcal{L}_{\infty}$ space. Therefore, all we have to do is to prove the case $n=2$. Let $X_{1}$ and $X_{2}$ be $\mathcal{L}_{\infty}$ spaces, let $Y_{1}$ and $Y_{2}$ be $\mathcal{L}_{1}$ spaces, and let $u_{j}: X_{j} \rightarrow Y_{j}$ be a linear operator, $j=1,2$. As in Proposition 3.4, we have to prove that $S=T\left(u_{1}, u_{2}\right): X_{1} \times X_{2} \rightarrow \mathbf{K}$ is integral for every $T \in \mathcal{L}^{2}\left(Y_{1}, Y_{2}\right)$. This is equivalent to prove that the associated linear operator $S_{1}: X_{1} \rightarrow X_{2}^{*}$ is integral. Now. we have the decomposition $S_{1}=u_{2}^{*}$ o 
$T_{1} \circ u_{1}$. By Grothendieck's theorem [12. Theorem 3.7]. $u_{1}$ and $u_{2}^{*}$ are 2-summing. Then, [12, Theorem 2.22] tells us that $S_{1}$ is 1 -summing and therefore integral [28, Theorem III.3].

It must be noticed that this argument gives also the case $n=2$ of Proposition 3.4. However, the general case cannot be obtained by this simple induction reasoning since GT and cotype 2 spaces are not stable under projective tensor products. In fact, by [26, Theorem 10.6], there exists a GT space $X$ with cotype 2 such that $X \widehat{\otimes}_{\pi} X=X \widehat{\otimes}_{\varepsilon} X$. By [16, Remark 1] and [12. Theorem 14.1], this implies that $X \widehat{\otimes}_{\pi} X$ does not have finite cotype and therefore (see [26, Corollary 6.13$]$ and $[12$, Theorem 14.5]) $X \widehat{\otimes}_{\pi} X$ cannot be a GT space.

Proposition 3.1 also allows us to give a new multilinear characterization of $\mathcal{L}_{\infty}$ spaces.

Proposition 3.6. Given $X_{1} \ldots X_{n}$ Banach spaces, the following are equivalent:

(i) $X_{1}, \ldots, X_{n}$ are $\mathcal{L}_{\infty}$ spaces;

(ii) for every Banach space $Y$ and for every multiple 1-summing $n$-linear operator $T: X_{1} \times \ldots \times X_{n} \rightarrow Y$, we have that $T$ is integral.

Proof. To see that (ii) implies (i) we consider an arbitrary Banach space $Y$ and an arbitrary absolutely summing linear operator $u: X_{1} \rightarrow Y$. By [28. Theorem III.3], if we prove that $u$ is integral, we will obtain that $X_{1}$ is an $\mathcal{L}_{x}$ space (we reason identically for $2 \leq j \leq n)$. For $2 \leq j \leq n$ we consider $x_{j} \in B_{X_{j}}$ and $x_{j}^{*} \in B_{X_{j}^{*}}$ such that $x_{j}^{*}\left(x_{j}\right)=1$. It is trivial that $T=u \& x_{2}^{*} \& \ldots 8 x_{n}^{*}: X_{1} \times \ldots \times X_{n} \rightarrow Y$ is multiple 1summing. Using the hypothesis, we have that $\widehat{T}: X_{1} \widehat{\&}_{\varepsilon} \ldots \widehat{\otimes}_{\varepsilon} X_{n} \rightarrow Y$ is integral. and so is $u=\widehat{T} v$, where $v: X_{1} \rightarrow X_{1} \widehat{\otimes}_{\varepsilon} \ldots \widehat{\otimes}_{\varepsilon} X_{n}$ is given by $v\left(x_{1}\right)=x_{1} \& \ldots \& x_{n}$.

To see that (i) implies (ii), we reason for the case $n=2$ (the general case can be obtained similarly by induction). Choose a bilinear operator $T: X_{1} \times X_{2} \rightarrow Y$, and let $T_{1}: X_{1} \rightarrow \mathcal{L}\left(X_{2}, Y\right)$ be its associated linear operator. Using standard localization arguments we can deduce from Proposition 3.1 that, if $T \in \Pi_{1}^{2}\left(X_{1}, X_{2}: Y\right)$, then $T_{1} \in \Pi_{1}\left(X_{1}, \Pi_{1}\left(X_{2}, Y\right)\right)$. Now, [28, Theorem III.3] tells us that $T_{1} \in I\left(X_{1}, I\left(X_{2}, Y\right)\right)$ and, by [30], we can conclude that $T$ is integral.

Remark 3.7. Since 1-dominated multilinear operators (see [20] for definition and basic facts) are easily seen to be multiple 1-summing. Theorem 3.6 is weaker in one direction and stronger in the other direction than the main result in [9].

Next we are going to prove our main result relating multiple $(p, 1)$-summing multilinear operators with the $p$-variation of their representing polymeasure. 
Theorem 3.8. Let $\left(\Omega_{j}, \Sigma_{j}\right), 1 \leq j \leq n$, be measurable spaces, let $1 \leq p<\infty$ and let $Y$ be a Banach space. Consider a multilinear operator $T: B\left(\Sigma_{1}\right) \times \ldots \times B\left(\Sigma_{n}\right) \rightarrow$ $Y$ with representing polymeasure $\gamma: \Sigma_{1} \times \ldots \times \Sigma_{n} \rightarrow Y$. Then $T$ is multiple $(p .1)$ summing if and only if $v_{p}(\gamma)<\infty$. Moreover, in that case

$$
v_{p}(\gamma) \leq \pi_{(p, 1)}(T) \leq \begin{cases}2^{n(1-1 / p)} v_{p}(\gamma) & \text { in the real case } \\ 2^{n(2-1 / p)} v_{p}(\gamma) & \text { in the complex case }\end{cases}
$$

Proof. Let us first suppose that $T$ is multiple $(p, 1)$-summing and let us consider $\Sigma_{j}$-partitions $\left(A_{k_{j}}^{j}\right)_{k_{j}=1}^{r_{j}}$ of $\Omega_{j}, 1 \leq j \leq n$. For every $\mu_{j} \in B\left(\Sigma_{j}\right)^{*}$ with $\left\|\mu_{j}\right\| \leq 1$ we have $\sum_{k_{j}=1}^{r_{j}}\left|\mu_{j}\left(A_{k_{j}}^{j}\right)\right| \leq 1$. Therefore

$$
\begin{aligned}
\left(\sum_{k_{1}=1}^{r_{1}} \ldots \sum_{k_{n}=1}^{r_{n}}\left\|\gamma\left(A_{k_{1}}^{1}, \ldots, A_{k_{n}}^{n}\right)\right\|^{p}\right)^{1 / p} & =\left(\sum_{k_{1}=1}^{r_{1}} \ldots \sum_{k_{n}=1}^{r_{n}}\left\|T\left(\chi_{A_{k_{1}}^{1}}, \ldots, \chi_{A_{k_{n}}^{n}}\right)\right\|^{p}\right)^{1 / p} \\
& \leq \pi_{(p, 1)}(T)
\end{aligned}
$$

We now prove the converse in the real case. the complex case follows easily considering real and imaginary parts. Using density; it is enough to check for sequences in $S\left(\Sigma_{i}\right)$. So, let $\left(f_{i_{j}}^{j}\right)_{i_{j}=1}^{m_{j}} \subset S\left(\Sigma_{j}\right), 1 \leq j \leq n$. There exist $\Sigma_{j}$-partitions $\left(A_{k_{j}}^{j}\right)_{k_{j}=1}^{r_{j}}$ of $\Omega_{j}, 1 \leq j \leq n$, and real numbers $a_{i_{j}, k_{j}}^{j}$ such that

$$
f_{i_{j}}^{j}=\sum_{k_{j}=1}^{r_{j}} a_{i_{j}, k_{j}}^{j} \chi_{A_{k_{j}}^{j}}
$$

Claim 1. The norm $\left\|\left(f_{i_{j}}^{j}\right)_{i_{j}=1}^{m_{j}}\right\|_{1}^{\omega} \leq 1$ if and only if $\left\|a_{j}\right\| \leq 1$, where $a_{j}: l_{1}^{r_{j}} \rightarrow l_{1}^{m_{j}}$ is the operator defined by

$$
a_{j}\left(e_{k_{j}}\right)=\sum_{i_{j}=1}^{m_{j}} a_{i_{j}, k_{j}}^{j} e_{i_{j}}
$$

Proof of the claim. Let us first suppose that $\left\|\left(f_{i_{j}}^{j}\right)_{i_{j}=1}^{m_{j}}\right\|_{1}^{\omega_{i}} \leq 1$, and consider $\left(c_{k_{j}}\right)_{k_{j}=1}^{r_{j}} \in B_{l_{1}^{r_{j}}}$. For each $1 \leq k_{j} \leq r_{j}$, choose $\omega_{k_{j}} \in A_{k_{j}}^{j}$ and let $\mu_{j}=\sum_{k_{j}=1}^{r_{j}} c_{k_{j}} \delta_{\omega_{k_{j}}}$, where $\delta_{\omega_{k_{j}}}$ is the evaluation at $\omega_{k_{j}}$. Then $\mu_{j} \in B_{B\left(\Sigma_{j}\right)^{*}}$ and

$$
\left\|a_{j}\left(\left(c_{k_{j}}\right)_{k_{j}=1}^{r_{j}}\right)\right\|=\sum_{i_{j}=1}^{m_{j}}\left|\sum_{k_{j}=1}^{r_{j}} c_{k_{j}} a_{i_{j}, k_{j}}^{j}\right|=\sum_{i_{j}=1}^{m_{j}}\left|\mu_{j}\left(f_{i_{j}}^{j}\right)\right| \leq 1
$$

which finishes this part of the proof. 
For the converse, suppose that $\left\|a_{j}\right\| \leq 1$ and choose $\mu_{j} \in B_{B\left(\Sigma_{j}\right)^{*}}$. Clearly

$$
\sum_{k_{j}=1}^{r_{j}}\left|\mu_{j}\left(A_{k_{j}}^{j}\right)\right| \leq 1
$$

and we get

$$
\sum_{i_{j}=1}^{m_{j}}\left|\mu_{j}\left(f_{i_{j}}^{j}\right)\right|=\sum_{i_{j}=1}^{m_{j}}\left|\sum_{k_{j}=1}^{r_{j}} a_{i_{j}, k_{j}}^{j} \mu_{j}\left(A_{k_{j}}^{j}\right)\right|=\left\|a_{j}\left(\left(\mu_{j}\left(A_{k_{j}}^{j}\right)\right)_{k_{j}=1}^{r_{j}}\right)\right\| \leq 1 .
$$

which finishes the proof of the claim.

We consider now the (non-linear) mapping

$$
F: \mathcal{L}\left(l_{1}^{r_{1}}, l_{1}^{m_{1}}\right) \times \ldots \times \mathcal{L}\left(l_{1}^{r_{n}} \cdot l_{1}^{m_{n}}\right) \longrightarrow \mathbf{R}
$$

defined by

$$
F\left(c_{1}, \ldots, c_{n}\right)=\left(\sum_{i_{1}=1}^{m_{1}} \ldots \sum_{i_{n}=1}^{m_{n}}\left\|\sum_{k_{1}=1}^{r_{1}} \ldots \sum_{k_{n}=1}^{r_{n}} c_{i_{1}, k_{1}}^{1} \ldots c_{i_{n}, k_{n}}^{n} \gamma\left(A_{k_{1}}^{1} \ldots, A_{k_{n}}^{n}\right)\right\|^{p}\right)^{1 / p}
$$

where $c_{i_{j}, k_{j}}^{j}=\left\langle c_{j}\left(e_{k_{j}}\right), e_{i_{j}}\right\rangle$.

It is easy to see that $F$ is continuous and separately convex. Therefore, its maximum in the compact set $B_{\mathcal{L}\left(l_{1}^{r_{1}} . l_{1}^{m_{1}}\right)} \times \ldots \times B_{\mathcal{L}\left(l_{1}^{r_{n}} \cdot l_{1}^{m_{n}}\right)}$ is attained on the product of extremal points $\left(b_{1}, \ldots, b_{n}\right)$.

Claim 2. If $b_{j} \in \operatorname{ext} B_{\mathcal{L}\left(l_{1}^{r_{j}} . l_{1}^{m_{j}}\right)}$ then, for every $k_{j} \in\left\{1, \ldots, r_{j}\right\}$, there exist $i_{j}\left(k_{j}\right) \in\left\{1, \ldots, m_{j}\right\}$ and $\varepsilon_{k_{j}}^{j} \in\{1,-1\}$ such that $b_{i_{j}, k_{j}}^{j}=\varepsilon_{k_{j}}^{j} \delta_{i_{j}\left(k_{j}\right)}^{i_{j}}$. Obviously, $i_{j}\left(k_{j}\right)$ and $\varepsilon_{k_{j}}^{j}$ are unique.

Proof of the claim. If there is a $k_{j}^{0}$ such that $\left(b_{i_{j}, k_{j}{ }_{j}}\right)_{i_{j}=1}^{m_{j}}$ is not of the form $\varepsilon_{k_{j}^{0}} e_{i_{j}\left(k_{j}^{0}\right)}$, then $\left(b_{i_{j}, k_{j}^{0}}^{j}\right)_{i_{j}=1}^{m_{j}}$ is not an extremal point of $B_{l_{1}^{m_{j}}}$. Consequently, there exist two different sequences $\left(y_{i_{j}}^{j}\right)_{i_{j}=1}^{m_{j}} .\left(z_{i_{j}}^{j}\right)_{i_{j}=1}^{m_{j}} \in B_{l_{1} m_{j}}$ with $b_{i_{j}, k_{j}^{j}}^{j}=\frac{1}{2} y_{i_{j}}^{j}+\frac{1}{2} z_{i_{j}}^{j}$ for all $i_{j}=1, \ldots, m_{j}$.

By setting

$$
y_{i_{j}, k_{j}}^{j}=\left\{\begin{array}{ll}
b_{i_{j}, k_{j}}^{j}, & k_{j} \neq k_{j}^{0}, \\
y_{i_{j}}^{j}, & k_{j}=k_{j}^{0},
\end{array} \quad \text { and } \quad z_{i_{j}, k_{j}}^{j}= \begin{cases}b_{i_{j}, k_{j},}^{j}, & k_{j} \neq k_{j}^{0}, \\
z_{i_{j}}^{j}, & k_{j}=k_{j}^{0}\end{cases}\right.
$$


we have that $b_{i_{j}, k_{j}}^{j}==\frac{1}{2} y_{i_{j}, k_{j}}^{j}+\frac{1}{2} z_{i_{j}, k_{j}}^{j}$ for every $i_{j}, k_{j}$, that $\left(y_{i_{j}, k_{j}}^{j}\right)_{i_{j}, k_{j}} \neq\left(z_{i_{j}, k_{j}}^{j}\right)_{i_{j}, k_{j}}$ and that $\left(y_{i_{j}, k_{j}}^{j}\right)_{i_{j}, k_{j}},\left(z_{i_{j}, k_{j}}^{j}\right)_{i_{j}, k_{j}} \in B_{\mathcal{L}\left(l_{1}^{r_{j}}, l_{l}^{m_{j}}\right)}$. In conclusion. $b_{j}$ is not extremal, which finishes the proof of the claim.

So, we have

$$
\begin{aligned}
& \left(\sum_{i_{1}=1}^{m_{1}} \ldots \sum_{i_{n}=1}^{m_{n}}\left\|T\left(f_{i_{1}}^{1}, \ldots, f_{i_{n}}^{n}\right)\right\|^{p}\right)^{1 / p} \\
& =F\left(a_{1}, \ldots, a_{n}\right) \\
& \leq F\left(b_{1}, \ldots, b_{n}\right) \\
& =\left(\sum_{i_{1}=1}^{m_{1}} \ldots \sum_{i_{n}=1}^{m_{n}}\left\|\sum_{\left\{k_{1}: i_{1}\left(k_{1}\right)=i_{1}\right\}} \ldots \sum_{\left\{k_{n}: i_{n}\left(k_{n}\right)=i_{n}\right\}} \varepsilon_{k_{1}}^{1} \ldots \varepsilon_{k_{n}}^{n} \gamma_{\left(m_{1}\right.}\left(A_{k_{1}}^{1} \ldots, A_{k_{n}}^{n}\right)\right\|^{p}\right)^{1 / p} \\
& \left.\leq\left(\sum_{i_{1}=1}^{m_{1}} \ldots \sum_{i_{n}=1}^{m_{n}}\left(\sum_{\phi \in \Phi} \| \sum_{k_{1} \in \Gamma_{i_{1}, \rho(1)}^{1}} \ldots \sum_{k_{n} \in \Gamma_{i_{n} . \circ(n)}^{n}} \gamma_{\left(A_{k_{3}}\right.}^{1} \ldots, A_{k_{n}}^{n}\right) \|\right)^{p}\right)^{1 / p}
\end{aligned}
$$

with

$$
\begin{aligned}
& \Gamma_{i_{j,+}}^{j}=\left\{k_{j}: i_{j}\left(k_{j}\right)=i_{j} \text { and } \varepsilon_{k_{j}}^{j}=1\right\} . \\
& \Gamma_{i_{j,-}}^{j}=\left\{k_{j}: i_{j}\left(k_{j}\right)=i_{j} \text { and } \varepsilon_{k_{j}}^{j}=-1\right\} .
\end{aligned}
$$

and $\Phi$ the set of mappings from $\{1 \ldots . n\}$ to $\{+,-\}$.

We note by $B_{i_{j}, j}^{j}=\bigcup_{k_{j} \in \Gamma_{i_{j},+}^{j}} A_{k_{j}}^{j}$ and by $B_{i_{j},-}^{j}=\bigcup_{k_{j} \in \Gamma_{i_{j}-}^{j}} A_{k_{j}}^{j}$. We have that. for each $j$, the sets $B_{i_{j},+}^{j}$ and $B_{i_{j,-}}^{j}$ are all disjoint. So,

$$
\begin{aligned}
& \left(\sum_{i_{1}=1}^{m_{1}} \ldots \sum_{i_{n}=1}^{m_{n}}\left\|T\left(f_{i_{1}}^{1}, \ldots, f_{i_{n}}^{n}\right)\right\|^{p}\right)^{1 / p} \\
& \leq\left(\sum_{i_{1}=1}^{m_{1}} \ldots \sum_{i_{n}=1}^{m_{n}}\left(\sum_{\phi \in \Phi}\left\|_{k_{1} \in \Gamma_{i_{1}, \infty(1)}^{1}} \ldots \sum_{k_{n} \in \Gamma_{i_{n}, 0 i n}^{n}} \eta_{i}\left(A_{k_{1}}^{1} \ldots . A_{k_{n}}^{n}\right)\right\|\right)^{p}\right)^{1 / p} \\
& =\left(\sum_{i_{1}=1}^{m_{1}} \ldots \sum_{i_{n}=1}^{m_{n}}\left(\sum_{\delta \in \Phi}\left\|\gamma\left(B_{i_{1}, o(1)}^{1} \ldots . B_{i_{n}, o(n)}^{n}\right)\right\|\right)^{p}\right)^{1 / p} \\
& \leq 2^{n(1-1 / p)}\left(\sum_{i_{1}=1}^{m_{1}} \ldots \sum_{i_{n}=1}^{m_{n}} \sum_{\phi \in \Phi}\left\|i_{i}\left(B_{i_{1} . \circ(1)}^{1} \ldots B_{i_{n} . \phi(n)}^{n}\right)\right\|^{p}\right)^{1 / p} \\
& \leq 2^{n(1-1 / p)} v_{p}(\gamma) .
\end{aligned}
$$

Using Theorems 2.3 and 3.8 and the comments abore about polymeasures. it is very easy to obtain the $C(K)$ version of Theorem 3.8 . 
Theorem 3.9. Let $K_{j}$ be compact Hausdorff spaces, $Y$ a Banach space and $T: C\left(K_{1}\right) \times \ldots \times C\left(K_{n}\right) \rightarrow Y$ be a multilinear operator with representing polymeasure $\gamma: \Sigma_{1} \times \ldots \times \Sigma_{n} \rightarrow Y^{* *}$. Then, $T$ is multiple $(p, 1)$-summing if and only if $v_{p}(\gamma)<\infty$.

Moreover, in this case,

$$
v_{p}(\gamma) \leq \pi_{(p, 1)}(T) \leq \begin{cases}2^{n(1-1 / p)} v_{p}(\gamma) & \text { in the real case } \\ 2^{n(2-1 / p)} v_{p}(\gamma) & \text { in the complex case }\end{cases}
$$

Remark 3.10. The case $n=1$ of Theorem 3.9 gives a new characterization of $(p, 1)$-summing linear operators from $C(K)$ spaces in terms of their representing measure.

As a corollary, we obtain a new proof of a classical result ([21, p. 14]).

Corollary 3.11. Let $K$ be a compact Hausdorff space, $p \geq 1$ and $Y$ be a Banach space. A linear operator $T: C(K) \rightarrow Y$ is $(p, 1)$-summing if and only if

$$
\sup \left\{\left(\sum_{i=1}^{m}\left\|T\left(f_{i}\right)\right\|^{p}\right)^{1 / p}:\left(f_{i}\right)_{i=1}^{m} \in B_{C(K)} \text { with disjoint supports }\right\}<\infty .
$$

Proof. First of all, it should be noticed that. if $\left(f_{i}\right)_{i=1}^{m}$ have disjoint supports, then $\left\|\left(f_{i}\right)_{i=1}^{m}\right\|_{1}^{\omega}=\max _{1 \leq i \leq m}\left\|f_{i}\right\|$. So, by Theorem 3.9 , it is enough to see that $v_{p}(\gamma)$ is less than or equal to (2), where $\gamma: \Sigma \rightarrow Y^{* *}$ is the representing measure of $T$. The proof of this fact for $p=1$ can be seen in [13, p. 163]. The general case can be obtained with obvious modifications.

Remark 3.12. The constant $2^{n(1-1 / p)}$ in the real case for Theorems 3.8 and 3.9 is optimal. To see this, we can consider $T: l_{\infty}^{2} \times \ldots \times l_{\infty}^{2} \rightarrow \mathbf{R}$ given by $T\left(\left(x_{1}, y_{1}\right), \ldots,\left(x_{n}, y_{n}\right)\right)=\prod_{j=1}^{n}\left(y_{n}-x_{n}\right)$. In the complex case, however, we do not know (even in the case $n=1$ ) what the optimal constant is.

It is now a natural question whether we can obtain a result similar to Proposition 3.1 for multiple $(p, 1)$-summing multilinear operators. The answer is no and the clue is [5, Theorem 3.2] (see Theorem 3.17 below).

Example 3.13. Let $X, Y$ and $Z$ be infinite-dimensional $\mathcal{L}_{\infty}$ spaces. Then we have that

$$
\Pi_{(2,1)}\left(X, \Pi_{(2,1)}\left(Y, Z^{*}\right)\right) \varsubsetneqq \Pi_{(2,1)}^{2}\left(X, Y: Z^{*}\right) .
$$


Proof. Using a version of Grothendieck's theorem ([12, Theorem 3.7]), we know that $\Pi_{(2,1)}\left(Y, Z^{*}\right)$ is isomorphic to $\left(Y \otimes_{\pi} Z\right)^{*}$. Moreover, it follows from Dvoretzki's theorem that, for any $\varepsilon>0, Y^{*} \widehat{\otimes}_{\varepsilon} Z^{*}$ contains the $l_{\infty}^{n}$ 's, $(1+\varepsilon)$-uniformly (see $[16$, Remark 1]). Since $Y^{*} \widehat{\otimes}_{\varepsilon} Z^{*}$ is isometrically embedded into $\left(Y \widehat{\otimes}_{\pi} Z\right)^{*}$, we get that $\left(Y \widehat{\otimes}_{\pi} Z\right)^{*}$ contains the $l_{\infty}^{n}$ 's, $(1+\varepsilon)$-uniformly (complemented).

Let $i_{n}: l_{\infty}^{n} \hookrightarrow\left(Y \otimes_{\pi} Z\right)^{*}, p_{n_{2}}:\left(Y \otimes_{\pi} Z\right)^{*} \rightarrow l_{\infty}^{n}$ be such that $p_{n} i_{n}=\operatorname{Id}_{l_{\infty}^{n}} ;\left\|i_{n}\right\|=1$ and $\left\|p_{n}\right\| \leq 2$. Let $X$ be an $\mathcal{L}_{\infty, \lambda}$ space, then, for every $n \in \mathbf{N}$, we can consider projections $R_{n}: X \rightarrow l_{\infty}^{n}$ with $\left\|R_{n}\right\| \leq \lambda$ and $\pi_{(2,1)}\left(R_{n}\right) \geq \sqrt{n}$.

For every $n \in \mathbf{N}$, we consider the operator $T_{n}=i_{n} R_{n}: X \rightarrow\left(Y \otimes_{\pi} Z\right)^{*}$ and its associated bilinear operator $\bar{T}_{n}: X \times Y \rightarrow Z^{*}$. Since $Z^{*}$ is an $\mathcal{L}_{1}$ space, it has cotype 2 . So, [5, Theorem 3.2] tells us that there exists $C>0$ such that, for every $n \in \mathbf{N}$,

$$
\pi_{(2,1)}\left(\bar{T}_{n}\right) \leq C\left\|T_{n}\right\| \leq \lambda C
$$

As $\pi_{(2,1)}\left(R_{n}\right) \geq \sqrt{n}$, we have that $\pi_{(2,1)}\left(T_{n}\right)=\pi_{(2,1)}\left(i_{n} R_{n}\right) \geq \frac{1}{2} \sqrt{n}$. This proves the non-equivalence of the corresponding norms, and, hence, the existence of an operator $T \in \Pi_{(2,1)}^{2}\left(X, Y ; Z^{*}\right)$ such that its associated operator $T_{1}: X \rightarrow \Pi_{(2,1)}\left(Y, Z^{*}\right)$ is not $(2,1)$-summing.

To give an explicit counterexample, let $X=c_{0} . Y=Z=l_{\infty}$. Then $\left(Y \widehat{\otimes}_{\pi} Z\right)^{*}$ contains an isomorphic copy of $c_{0}$ (see [1]), and we can consider $T: X \times Y \rightarrow Z^{*}$ as the bilinear operator associated to $T_{1}: c_{0} \hookrightarrow\left(Y \widehat{\otimes}_{\pi} Z\right)^{*}$.

Remark 3.14. In fact, if we use the multilinear version of Grothendieck's theorem given in [5, Theorem 3.1] instead of [5, Theorem 3.2], we can prove, with the same argument, the existence of a multiple 2-summing bilinear operator $T: X \times Y \rightarrow$ $Z$ such that $T_{1} \notin \Pi_{2}\left(X, \Pi_{2}(Y, Z)\right)$, solving a question stated in [27].

Now, we are going to extend, to the multilinear setting, another linear property that extends the field of applications of the above results. First we need a proposition, whose proof follows immediately from the definitions.

Proposition 3.15. Let $T: X_{1} \times \ldots \times X_{n} \rightarrow Y$ be a multilinear operator and let $1 \leq p_{1}, \ldots, p_{n} \leq q<\infty$. The following are equivalent:

(i) $T$ is multiple $\left(q ; p_{1}, \ldots, p_{n}\right)$-summing;

(ii) there exists a constant $K>0$ such that for every $m_{2}, \ldots, m_{n} \in \mathbf{N}$ and every choice of sequences $\left(x_{i_{j}}^{j}\right)_{i_{j}=1}^{m_{j}} \subset X_{j}$, with $\left\|\left(x_{i_{j}}^{j}\right)_{i_{j}=1}^{m_{j}}\right\|_{p_{j}}^{w_{j}} \leq 1,2 \leq j \leq n$, we have that the associated linear operator

$$
S: X_{1} \longrightarrow l_{q}^{m_{2} \ldots m_{n}}(Y)
$$

given by

$$
S\left(x_{1}\right)=\left(T\left(x_{1}, x_{i_{2}}^{2}, \ldots, x_{i_{n}}^{n}\right)\right)_{i_{2} \ldots \ldots i_{n}=1}^{m_{2} \ldots \ldots m_{n}}
$$


is $\left(q, p_{1}\right)$-summing and it satisfies

$$
\pi_{\left(q \cdot p_{1}\right)}(S) \leq K
$$

In this case, $\pi_{\left(q: p_{1} \ldots . p_{n}\right)}(T)=\min \{K: K$ satisfies $(3)\}$

Proposition 3.16. Let $1 \leq p_{1}, \ldots, p_{n}<q<x$ and let $K_{1}, \ldots, K_{n}$ be compact Hausdorff spaces. A multilinear operator $T: C\left(K_{1}\right) \times \ldots \times C\left(K_{n}\right) \rightarrow Y$ is multiple $(q, 1)$-summing if and only if it is multiple $\left(q: p_{1} \ldots p_{n}\right)$-summing.

Proof. We reason in the bilinear case, the reasonings being similar in the general case. Suppose $T$ : $C\left(K_{1}\right) \times C\left(K_{2}\right) \rightarrow Y$ is multiple $(q .1)$-summing. Then, for any sequence $\left(x_{i_{1}}^{1}\right)_{i_{1}=1}^{m_{1}} \subset C\left(K_{1}\right)$ such that $\left\|\left(x_{i_{1}}^{1}\right)_{i_{1}=1}^{m_{1}}\right\|_{i} \leq 1$. the operator $S: C\left(K_{2}\right) \rightarrow l_{q}^{m}(Y)$ defined as in Proposition 3.15 is (q.1)-summing and satisfies

$$
\pi_{(q .1)}(S) \leq \pi_{(q .1)}(T)
$$

Let $i: l_{q}^{m}(Y) \rightarrow l_{q}(Y)$ be the natural inclusion. Applying [12, Theorem 10.9] to $i \circ S$, and using the injectivity of the operator ideal of the $(q, p)$ summing operators. we get that $S$ is $\left(q, p_{2}\right)$-summing and that

$$
\pi_{\left(q \cdot p_{2}\right)}(S) \leq K \pi_{(q \cdot 1)}(T)
$$

where the constant $K$ does not depend on the choice of $\left(x_{i_{1}}^{1}\right)_{i_{1}=1}^{m_{1}}$.

Therefore, $T$ is multiple $\left(q: 1, p_{2}\right)$-summing. We choose now any sequence $\left(x_{i_{2}}^{2}\right)_{i_{2}=1}^{m_{2}} \subset C\left(K_{2}\right)$ such that $\left\|\left(x_{i_{2}}^{2}\right)_{i_{2}=1}^{m_{2}}\right\|_{p_{2}}^{\dot{w}_{1}} \leq 1$ and reason similarly.

To end the paper, we are going to state some results concerning the $p$-variation of polymeasures. The starting point is [5. Theorem 3.2], which says the following.

Theorem 3.17. Let $X_{j}$ be a Banach space for $1 \leq j \leq n$ and let $Y$ be a cotype $q$ space. Then, every multilinear operator $T: X_{1} \times \ldots \times X_{n} \rightarrow Y$ is multiple $(q, 1)$ summing and

$$
\pi_{(q .1)}(T) \leq C_{q}(Y)^{n}\|T\| .
$$

where $C_{q}(Y)$ is the cotype $q$ constant of $Y$.

Using this result, the proof of the following surprising corollary is trivial.

Corollary 3.18. Let $Y$ be a cotype $q$ space and $\eta_{\eta}: \Sigma_{1} \times \ldots \times \Sigma_{n} \rightarrow Y$ be a polymeasure of bounded semivariation. Then $v_{q}(\gamma) \leq C_{q}(Y)^{n}\|\gamma\|$. In particular, every scalar polymeasure of bounded semivariation has bounded 2 -variation.

Note that, in general, scalar polymeasures do not have bounded variation (see $[7]$ ). 
We can improve the scalar case of the last two results. To this end, we consider the following classical theorem (see [4]: [10]. [17], [18]).

Theorem 3.19. (Littlewood-Bohnenblust-Hille) If $T$ is a continuous $n$-linear form on $c_{0}$, then

$$
\left(\sum_{i_{1}=1}^{\infty} \ldots \sum_{i_{n}=1}^{\infty}\left|T\left(e_{i_{1}}^{1}, \ldots, e_{i_{n}}^{n}\right)\right|^{2 n /(n+1)}\right)^{(n+1) / 2 n} \leq 2^{(n-1) / 2}\|T\| .
$$

This theorem allows us to prove the following result.

Corollary 3.20. Let $X_{1}, \ldots, X_{n}$ be Banach spaces. Every $n$-linear form $T: X_{1} \times \ldots \times X_{n} \rightarrow \mathbf{K}$ is multiple $(2 n /(n+1)$. 1$)$-summing and

$$
\pi_{(2 n /(n+1) .1)}(T) \leq 2^{(n-1) / 2}\|T\|
$$

Proof. Consider, for $1 \leq j \leq n$, sequences $\left(x_{i_{j}}^{j}\right)_{i_{j}=1}^{m_{j}} \subset X_{j}$ with $\left\|\left(x_{i_{j}}^{j}\right)_{i_{j}=1}^{m_{j}}\right\|_{1}^{\omega} \leq 1$. The operator $u_{j}: l_{\infty}^{m_{j}} \rightarrow X_{j}$ given by $u_{j}\left(e_{i_{j}}\right)=x_{i_{j}}^{j}$ satisfies

$$
\left\|u_{j}\right\|=\left\|\left(x_{i_{j}}^{j}\right)_{i_{j}=1}^{m_{j}}\right\|_{i} \leq 1 .
$$

We can now apply Theorem 3.19 to the multilinear operator

$$
S=T\left(u_{1}, \ldots, u_{n}\right): l_{\infty}^{m_{1}} \times \ldots \times l_{\infty}^{m_{n}} \longrightarrow \mathbf{K}
$$

to obtain that

$$
\begin{aligned}
& \left(\sum_{i_{1}=1}^{m_{1}} \ldots \sum_{i_{n}=1}^{m_{n}}\left|T\left(x_{i_{1}}^{1}, \ldots, x_{i_{n}}^{n}\right)\right|^{2 n /(n+1)}\right)^{(n+1) / 2 n} \\
& =\left(\sum_{i_{1}=1}^{m_{1}} \ldots \sum_{i_{n}=1}^{m_{n}}\left|S\left(e_{i_{1}}^{1}, \ldots, e_{i_{n}}^{n}\right)\right|^{2 n /(n+1)}\right)^{(n+1) / 2 n} \leq 2^{(n-1) / 2}\|S\| \leq 2^{(n-1) / 2}\|T\| .
\end{aligned}
$$

Finally, we have the following result.

Corollary 3.21. Every scalar polymeasure $\gamma: \Sigma_{1} \times \ldots \times \Sigma_{n} \rightarrow \mathbf{K}$ with bounded semivariation satisfies

$$
v_{2 n /(n+1)}(\gamma) \leq 2^{(n-1) / 2}\|\gamma\|
$$




\section{References}

1. Alaminos, J., Choi, Y.S., Kim, S. G. and PaYÁ. R., Norm attaining bilinear forms on spaces of contimuous functions, Glasgow Math. J. 40 (1998), 359-365.

2. Alencar, R., Multilinear mappings of nuclear and integral type, Proc. Amer. Math. Soc. 94 (1985), 33-38.

3. Aron, R. and Berner, P. D., A Hahn-Banach extension theorem for analytic mappings, Bull. Soc. Math. France 106 (1978), 3-24.

4. Bohnenblust, H. F. and Hille. E.. On the absolute convergence of Dirichlet series, Ann. of Math. 32 (1931), 600-622.

5. Bombal, F., Pérez-García, D. and Villaxleva. I.. Multilinear extensions of Grothendieck's theorem, to appear in Q. J. Math.

6. Bombal, F. and Villanleva, I., Multilinear operators on spaces of continuous functions, Funct. Approx. Comment. Math. 26 (1998), 117-126.

7. Bombal, F. and VillanueVA, I., Integral operators on the product of $C(K)$ spaces, J. Math. Anal. Appl. 264 (2001), 107-121.

8. Cabello Sánchez, F., García, R. and Villaxueva, I., Extension of multilinear operators on Banach spaces, in III Congress on Banach Spaces (Jarandilla de la Vera, 1998), Extracta Math. 15, pp. 291-334. Universidad de Extremadura, Badajoz, 2000.

9. Cilia, R., D'Anna, M. and Gutiérrez, J.. Polynomial characterization of $\mathcal{L}_{\infty}$ spaces, J. Math. Anal. Appl. 275 (2002). 900-912.

10. DAvie, A. M., Quotient algebras of uniform algebras. J. London Math. Soc. 7 (1973), $31-40$.

11. Defant, A. and Floret, K.. Tensor Norms and Operator Ideals, North-Holland Math. Studies, 176. North-Holland. Amsterdam, 1993.

12. Diestel, J., Jarchow, H. and Toxge, A., Absolutely Summing Operators, Cambridge Univ. Press, Cambridge, 1995.

13. Diestel, J. and Uhl, J. J., Vector Measures, Math. Surveys 15, Amer. Math. Soc., Providence, RI, 1977.

14. Dimant, V., Strongly p-summing multilinear operators, J. Math. Anal. Appl. 278 (2003), 182-193.

15. GonzÁlez, M. and GutiéRrez, J., The Dunford-Pettis property on tensor products, Math. Proc. Cambridge Philos. Soc. 131 (2001), 185-192.

16. John, K., Tensor powers of operators and nuclearity, Math. Nachr. 129 (1986), 115121.

17. KAIJSER, S., Some results in the metric theory of tensor products, Studia Math. 63 (1978), 157-170.

18. LitTlewood, J., On bounded bilinear forms in an infinite number of variables, $Q . J$. Math. 2 (1930), 167-171.

19. Matos, M. C., On multilinear mappings of nuclear type, Rev. Mat. Univ. Complut. Madrid 6 (1993), 61-81.

20. MATos, M. C., Fully absolutely summing and Hilbert-Schmidt multilinear mappings, Collect. Math 54 (2003), 111-1. 
21. Maurey, B., Type et cotype dans les espaces munis de structures locales inconditionelles, Séminaire Maurey-Schwartz 1973/74, Exp. Nos. 24 and 25, École Polytech., Paris, 1973.

22. Meléndez, Y. and Tonge, A.. Polynomials and the Pietsch domination theorem, Math. Proc. Roy. Irish Acad. 99A (1999). 195-212.

23. PÉREz-García, D., The inclusion theorem for multiple summing operators, Preprint.

24. PÉrez-García, D. and Villanceva, I., Multiple summing operators on Banach spaces, J. Math. Anal. Appl. 285 (2003), 86-96.

25. PÉrez-García, D. and Villanueva, I., A composition theorem for multiple summing operators, Preprint.

26. PISIER, G., Factorization of Linear Operators and Geometry of Banach Spaces, CBMS Regional Conf. Ser. in Math. 60. Amer. Math. Soc.. Providence, RI. 1986.

27. Ramanujan, M. S. and Schock, E., Operator ideals and spaces of bilinear operators. Linear and Multilinear Algebra 18 (1985). 307-318.

28. Retherford, J. R. and Stegall, C. P.. Fully nuclear and completely nuclear operators with applications to $\mathcal{L}_{1}$ - and $\mathcal{L}_{\infty}$-spaces, Trans. Amer. Math. Soc. 163 (1972), 457-492.

29. Rosenthal, H. P. and Szarek, S. J., On tensor products of operators from $L^{p}$ to $L^{q}$. in Functional Analysis (Austin. TX, 1987/1989) (Odell, E. and Rosenthal, H., eds.), Lecture Notes in Math. 1470, pp. 108-132, Springer-Verlag, BerlinHeidelberg, 1991.

30. Villanueva, I., Integral mappings between Banach spaces, J. Math. Anal. Appl. 279 (2003), 56-70.

David Pérez-García

Departamento de Análisis Matemático

Facultad de Matemáticas

Universidad Complutense de Madrid

ES-28040 Madrid

Spain

email: David_Perez@omat.ucm.es

Ignacio Villanueva

Departamento de Análisis Matemático

Facultad de Matemáticas

Universidad Complutense de Madrid

ES-28040 Madrid

Spain

email: ignaciova $\underline{a}$ mat.ucm.es 